\title{
Fluid-structure interaction analysis of rear spoiler vibration for energy harvesting potential
}

\author{
Mohammad Rasidi Rasani ${ }^{1,2 *}$, Mohammed Suleman Aldlemy ${ }^{1}$ and Zambri \\ Harun $^{1,2}$ \\ ${ }^{1}$ Department of Mechanical and Materials Engineering, Faculty of Engineering and \\ Built Environment, Universiti Kebangsaan Malaysia, 43600 Bangi, Selangor, Malaysia. \\ ${ }^{2}$ Centre for Automotive Research, Faculty of Engineering and Built Environment, \\ Universiti Kebangsaan Malaysia, 43600 Bangi, Selangor, Malaysia. \\ Phone: +60389216522; Fax: +60389118314 \\ *Email: rasidi@ukm.edu.my
}

\begin{abstract}
Increasing environmental and energy concerns constitutes an encouraging development of future automobiles and autonomous vehicles that utilize cleaner and renewable energy. Although primarily an aerodynamic device, car rear spoilers experience various vibrations and limited attention has been given to exploiting these vibrations for generating alternative energy. This paper aims to investigate the potential of using the flow-induced vibration of rear spoilers on automobiles for energy harvesting. To that end, a fluid-structure interaction analysis of an inverted NACA 2408 spoiler behind an Ahmed body was undertaken. An Arbitrary Lagrangian-Eulerian flow solver was coupled to a non-linear structural dynamic solver using a commercial software application. The vibrations of the rear spoiler placed at 0,50 and $100 \mathrm{~mm}$ below the top face of the Ahmed body under Reynolds number $\operatorname{Re}=2.7 \times 10^{6}$ were simulated. It was found that the vibration of the rear spoiler at the highest elevation showed the largest amplitudes and strain, but the vibration of the rear spoiler at the lowest elevation offers an extended period of vibration before reaching a steady state. With the rear spoiler positioned at the highest elevation, a vibration frequency of $70 \mathrm{~Hz}$ and a steady-state principal strain of $350 \mu \varepsilon$ may be achieved. These vibration levels compared well with previous investigation to sufficiently charge storage capacitors for wireless transmitters. The rear spoiler vibration may offer potential means for energy harvesting, and warrants further experiments under actual driving conditions.
\end{abstract}

Keywords: Fluid-structure interaction; energy harvesting; car spoiler; flow-induced vibration, piezoelectric.

\section{INTRODUCTION}

Environmental concerns and depleting fossil fuels are major issues that have influenced the automotive industry, including the development of renewable and alternative energy to either reduce the usage of traditional fuels (see for example, $[1,2]$ ) or to power various systems in vehicles. Future automobile solutions, including the development of autonomous vehicles, will require various monitoring and driving assistance systems that could be implemented via wireless electronics. However, current wireless systems are mostly battery powered, which requires frequent replacements [3] and may pose 
environmental hazards when disposed. Therefore, clean, low-cost and labour-free, alternative sources of energy have been the subject of active research in the past decade, especially in order to power wireless devices, such as sensor networks for monitoring various systems $[4,5]$. Among various concepts, harvesting energy from vibrations has gained much attention (see for example, [4-8]). Although attaching additional vibrationinduced energy harvesting components to a car may be feasible, a more elegant solution is by exploiting the use of the available components on the car. In road cars, the vibration from engine blocks $[9,10]$, wheel rotation [11] and vehicle suspension [12] have all been proposed to generate energy. However, to the best of the authors' knowledge, limited attention has been given to exploiting the rear spoilers or wings on cars for energy harvesting.

Functioning as an aerodynamic device, investigations on the rear spoilers or the wings on cars have often focused on aerodynamics and their flow characteristics. Tsai et al. [13] investigated the flow structures and aero-acoustic features generated in the various rear spoiler configurations of a passenger car, using computational flow dynamics. In another study, Kieffer et al. simulated a turbulent flow around the front and rear wing of a Formula Mazda race car and analyzed the effect of their angle of attack on car handling [14]. While, Jamei et al. analyzed the aerodynamic performance of a wing in close proximity to the ground, using both numerical and experimental means [15]. In a more recent study, Daryakenari et al. studied the effect of the rear spoilers on the aerodynamic characteristics of both a simple and a real road vehicle [16]. From the literature review, a limited investigation has focused on the vibration of rear vehicle spoilers, including harvesting energy from their vibrations. Therefore, in the present article, we aim to investigate the potential for exploiting the flow-induced vibrations of rear car spoilers for energy harvesting. To that end, we undertake the fluid-structure interaction (FSI) analysis of an inverted NACA 2408 rear spoiler and we employ an Ahmed body [17] with a $35^{\circ}$ rear slant angle in order to represent a car. An Ahmed body is a simplified generic bluff body that captures important aerodynamic features around conventional cars and has been frequently used as a reference test case in the automotive industry [18]. In order to further examine the influence of spoiler elevation on its flow-induced vibration amplitudes, the rear spoiler was placed at several positions in the transverse direction, while maintaining their streamwise position aligned with the rear face of the Ahmed body.

\section{MATERIALS AND METHODS}

\section{Computational Model}

Figure 1(a) depicts the computational domain that was used in all simulations. The Ahmed body and rear spoiler is enclosed in a computational domain of size $8 \mathrm{~L} \times 5 \mathrm{H} \times$ $5 W$ in the streamwise, transverse and spanwise directions, respectively (where $L=$ length, $H=$ height and $W=$ width of the Ahmed body). The size of the computational domain is consistent with the previous studies (see for example, [18-20]), where the blockage effects are minimized. The standard dimensions for the Ahmed body with the rear slant angle $\varphi$ $=35^{\circ}$ were used, as shown in Figure $1(\mathrm{~b})$, which may be found in $[17,18]$. Due to the complex geometry (i.e. Ahmed body in proximity to the rear spoiler) involved, the computational domain was discretised using unstructured tetrahedral elements. Finer grids were prescribed in the vicinity of the Ahmed body and of the rear spoiler, as highlighted in Figure 1(c), in order to accurately resolve wake features trailing both the Ahmed body and the rear spoiler. 


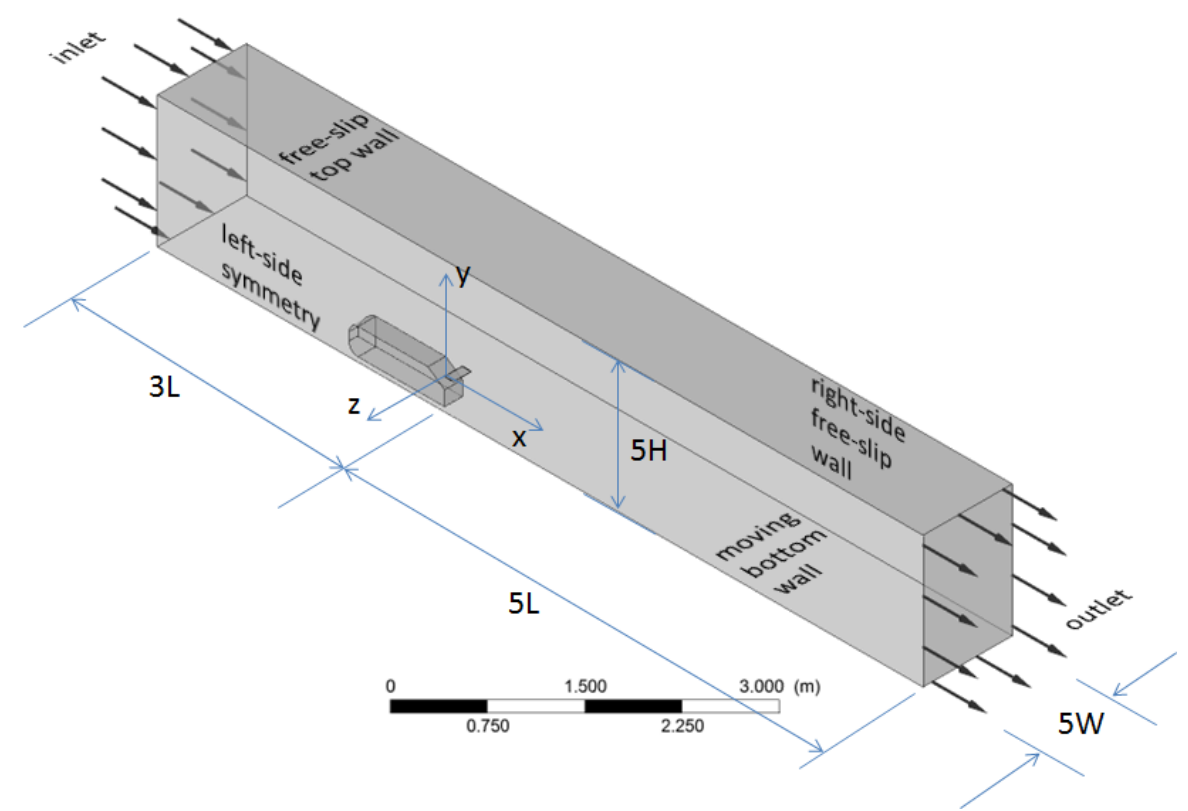

(a)
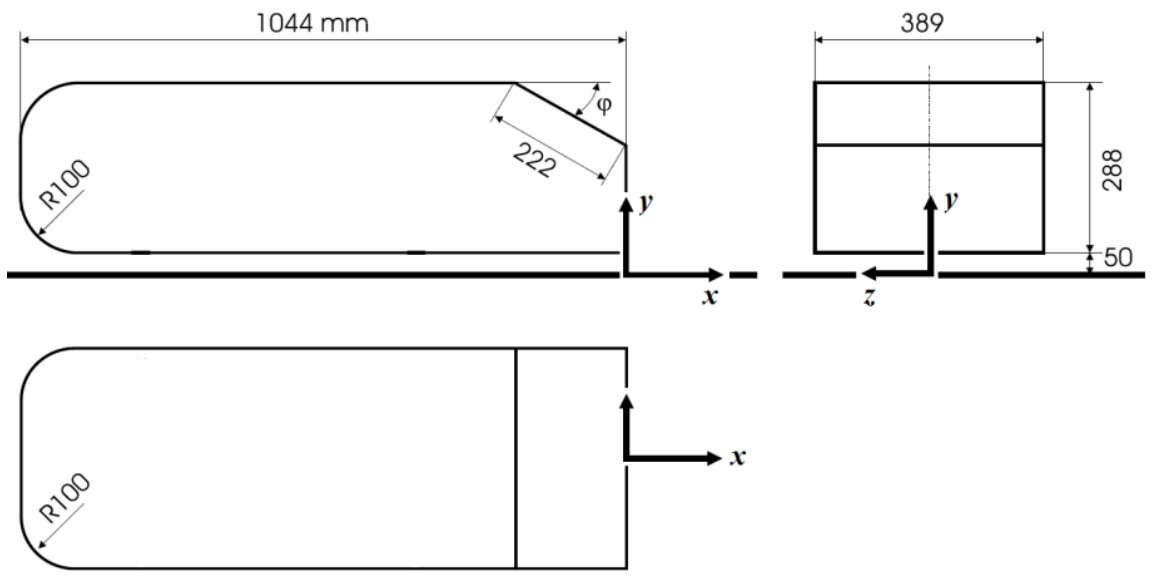

(b)

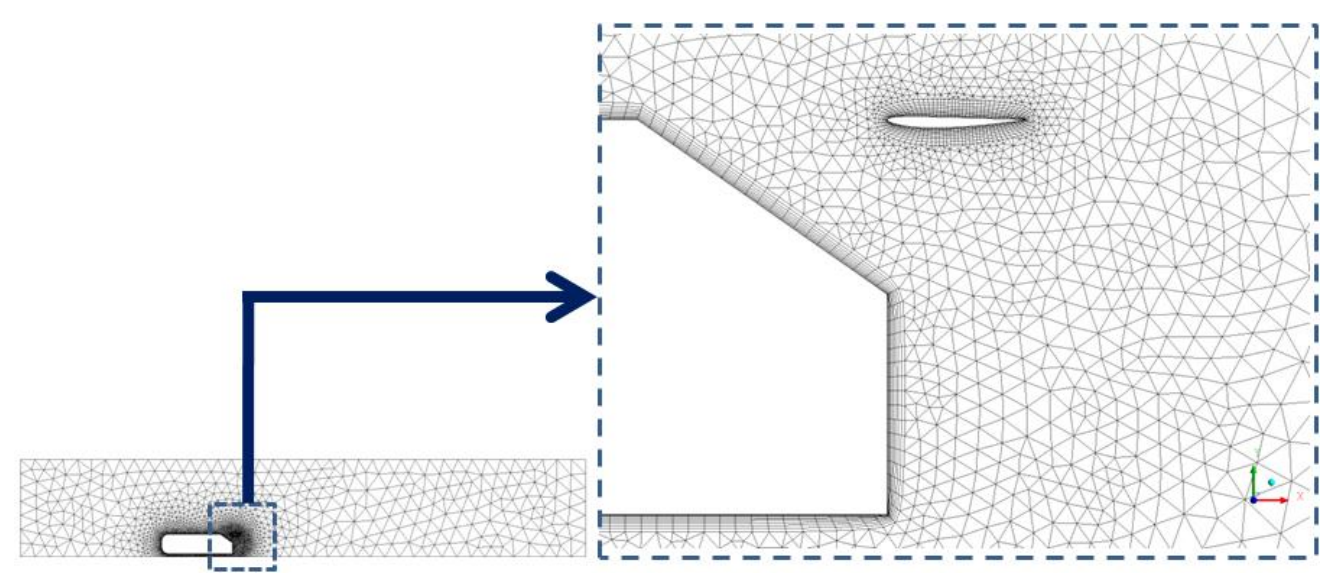

(c)

Figure 1. (a) Flow computational domain and boundary conditions; (b) Dimensions of the Ahmed body used in the present study (courtesy of [17, 18]); (c) Mesh on the flow model with inset showing the close-up of the mesh closer to the Ahmed body and rear spoiler. 
In addition, 10 layers of boundary layer elements that wrap the Ahmed body and the rear spoiler were defined in order to appropriately capture their boundary layer profile. The first layer height was specified such that their $y+$ values fall within the acceptable range for a log wall function employed in the turbulence model. The mesh were also refined in regions close to the Ahmed body and the rear spoilers, and in the wake region that is anticipated behind the Ahmed body, resulting in a total of 778,959 elements in the flow domain. In order to model the flow and to facilitate the fluid grid movement as the fluid boundary changes with the interfacing rear spoiler motion, the unsteady ReynoldsAveraged Navier-Stokes (RANS) equation is solved together with the conservation of mass, in an Arbitrary Lagrangian-Eulerian description [21]. Previous studies have shown that RANS-based models are still appropriate in comparison with the experimental results, for a rear slant angle of $35^{\circ}$ [20]. In the present study, we employ a $k$ - $\omega$ SST (shear stress transport) turbulence model in order to simulate the turbulent flow. In all cases, standard air properties were used, where the fluid density $\rho=1.185 \mathrm{~kg} / \mathrm{m}^{3}$ and the dynamic viscosity $\mu=1.831 \times 10^{-5} \mathrm{~kg} / \mathrm{ms}$. In order to facilitate validation, a uniform velocity of $40 \mathrm{~m} / \mathrm{s}$ was prescribed at the inlet, corresponding to the Reynolds number, $R e$ $=2.7 \times 10^{6}$ (based on length of Ahmed body). At the outlet, the boundary condition was prescribed to zero (atmospheric) pressure. A symmetric condition was imposed on the left-side boundary, while free-slip wall conditions were imposed on the top and right-side boundaries. The remaining boundaries representing the Ahmed body wall, rear spoiler wall and bottom wall, were set as no-slip wall boundary conditions.

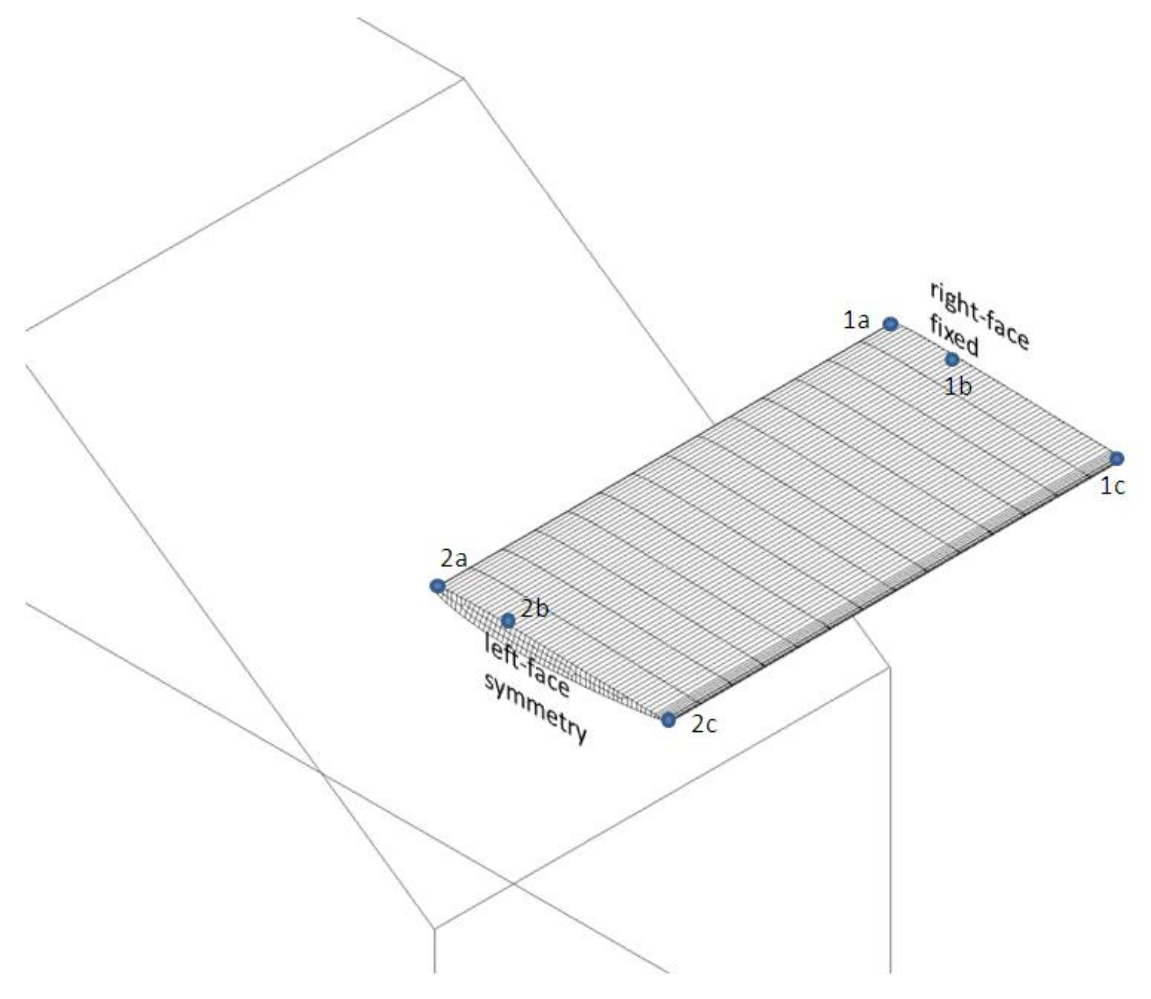

Figure 2. Rear spoiler geometry with corresponding discretization and boundary conditions. Points $[1 \mathrm{a}, 1 \mathrm{~b}, 1 \mathrm{c}]$ and $[2 \mathrm{a}, 2 \mathrm{~b}, 2 \mathrm{c}]$ represent the points near the fixed face and symmetric face, respectively (where ' $a$ ' denotes the point located along the leading edge, ' $b$ ' denotes the point located along the maximum camber and ' $c$ ' denotes the point located along the trailing edge of the spoiler). 


\section{Structural Model}

In the present study, we considered the inverted NACA 2408 profile [22] (with zero angle of attack) to represent our rear spoiler, where its chord length is $100 \mathrm{~mm}$ and its maximum camber of $2 \%$ is located at $30 \%$ of its chord. Figure 2 shows the mesh and boundary conditions imposed on the rear spoiler, where the right-face was assumed fixed and the left-face was set as a symmetric condition. At the same time, both the top and the bottom surface of the rear spoiler were subjected to the aerodynamic loads obtained from the flow solver. A total of 1680 solid elements were used to discretize the rear spoiler. In all cases, we considered our spoiler to be made of ABS material, where its elastic modulus, $E=2.2$ GPa and its Poisson ratio, $v=0.35$. A non-linear equation of motion considering large deformation was implemented for solving the dynamic response of the rear spoiler.

\section{Flow-Structure Coupling}

The interaction between flow and the rear spoiler is captured by imposing kinematic and dynamic compatibility at the common fluid-structure interface boundaries (i.e. at both top and bottom surfaces of the rear spoiler that are exposed to the flow behind the rear slant of the Ahmed body). These are the matching of the flow velocities with the spoiler velocities at the interfacing fluid-structure boundaries and the balance of stresses in the flow with stresses in the interfacing spoiler surface, respectively [23]. This implies that loading on the rear spoiler is obtained from the aerodynamic pressures and shear stresses computed from the flow solver. Although a fixed grid approach to modelling the flowstructure interaction, such as an immersed solid method [24] or an immersed boundary method [25], are computationally favourable in terms of avoiding the mesh regeneration efforts, this approach may lack an accurate flow resolution of the boundary layers around the structure. Therefore, in the present study, a dynamic grid approach is adopted instead. A commercial flow solver (ANSYS-CFX) is employed to solve the flow around the Ahmed body and the rear spoiler, which is then coupled to a commercial finite element solver (ANSYS-Mechanical) that is employed to solve the rear spoiler motion. During this partitioned coupling approach, the grids in the fluid domain dynamically deform according to the spoiler motion. In all simulations, a timestep size of $0.001 \mathrm{~s}$ was used, which was appropriate with the first mode frequency of the rear spoiler. Furthermore, in all cases, the unsteady flow-structure couplings were initiated from a steady state solution of the flow around a rigid rear spoiler and the Ahmed body.

\section{RESULTS AND DISCUSSION}

\section{Model Validation}

Flow around an Ahmed body has been reported in numerous investigations (see for example, $[17-20,26]$ ) at $R e=2.7 \times 10^{6}$ (based on length $L$ ) or $R e=768000$ (based on height $H$ ). Table 1 compiles comparisons of the drag coefficients around an Ahmed body with a $35^{\circ}$ rear slant angle between the present model and previous investigations. Although there is a slight discrepancy when considering stilts, it is estimated that this discrepancy is at most, within $19 \%$ of the experimental $C_{d}$.

In addition, Figures 3 and 4 present the pressure contours with streamlines and the velocity vectors with contours at several planes normal to the streamwise direction of the Ahmed body in the present model. Figure 3 indicates that the present model captures an appropriate pressure distribution around the Ahmed body, where the maximum pressure occurs at the front face as the velocity stagnates and the minimum pressure is generated nearing the top face as the flow accelerates. As the drag is generated significantly from 
the pressure difference around the Ahmed body, the favourable comparison between the present drag coefficient with previous studies suggests that reasonable pressure values are also captured in the present model. Figure 3(b) highlights the formation of a large wake region above a smaller wake region behind the rear face of the Ahmed body, similar with the findings in previous investigations (see for example, Figure 5 in ref. [19]).

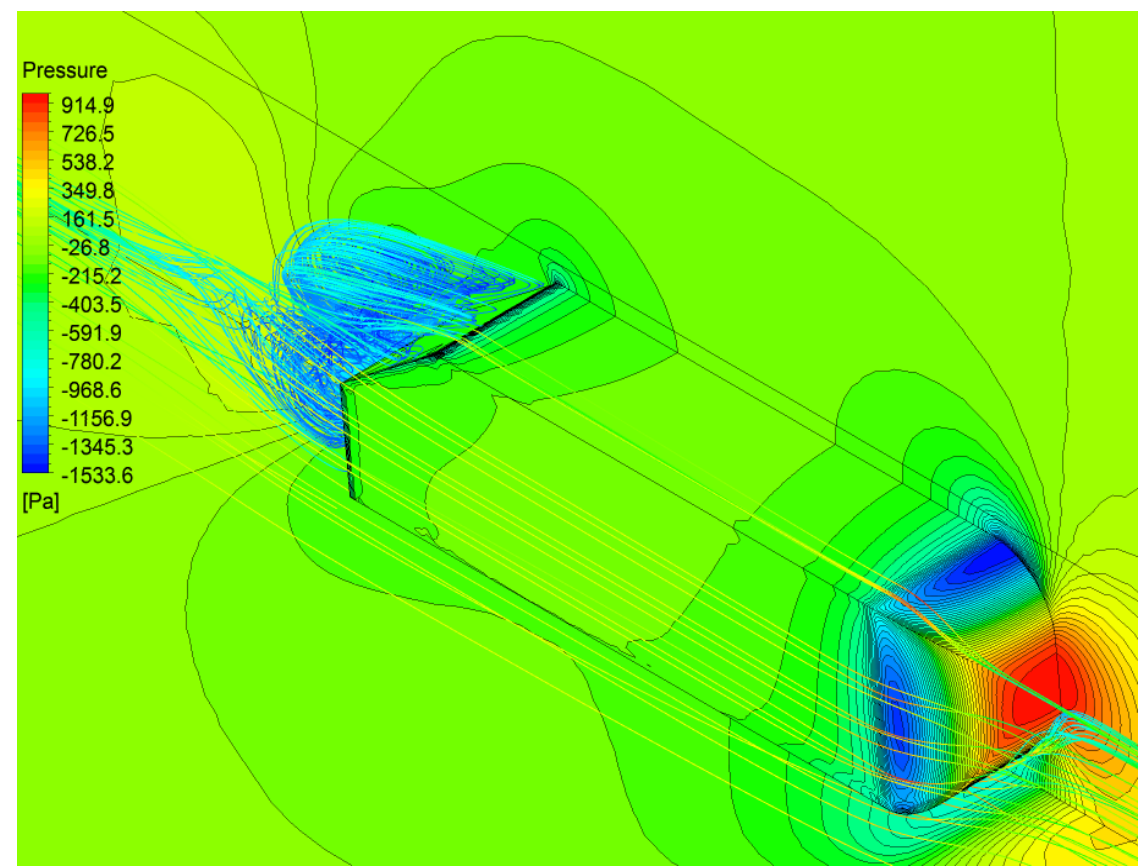

(a)

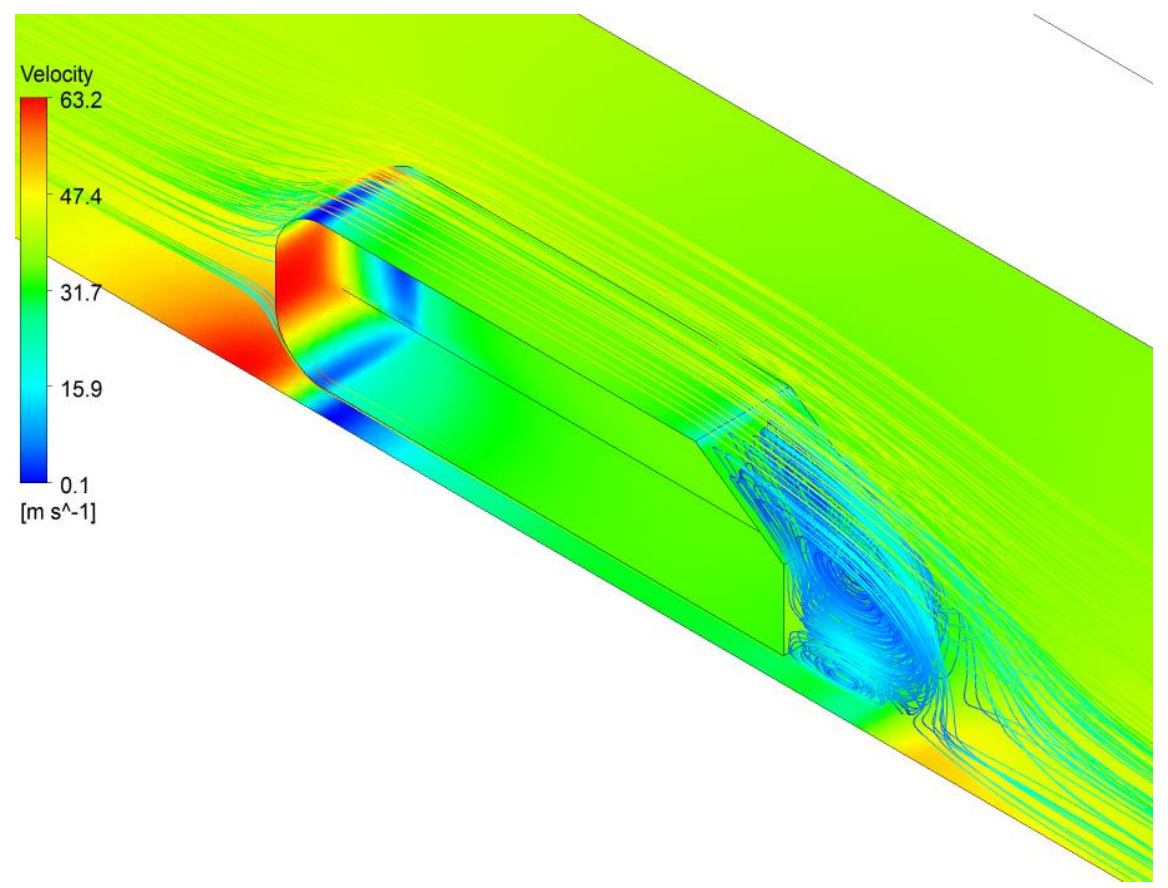

(b)

Figure 3. Pressure contour and streamlines isometric views (a) view from the right; (b) view from the left. 
Table 1. Coefficient of drag $\left(C_{d}\right)$ on an Ahmed body.

\begin{tabular}{ll}
\hline Case & $C_{d}$ \\
\hline Present study & $0.283^{\mathrm{a}}$ \\
SST $k-\omega[19]$ & $0.289^{\mathrm{a}}$ \\
SST $k-\omega[19]$ & $0.313^{\mathrm{b}}$ \\
EASM (explicit algebraic stress model) [19] & $0.236^{\mathrm{a}}$ \\
EASM [19] & $0.257^{\mathrm{b}}$ \\
Spallart Almaras [19] & $0.340^{\mathrm{a}}$ \\
Spallart-Almaras based DES (detached eddy simulation) [26] & $0.252^{\mathrm{a}}$ \\
Spallart-Almaras based RANS [26] & $0.319^{\mathrm{b}}$ \\
Experiment [17, 19] & $0.26^{\mathrm{b}}$ \\
\hline a
\end{tabular}

${ }^{\mathrm{a}}$ no stilts ${ }^{\mathrm{b}}$ with stilts

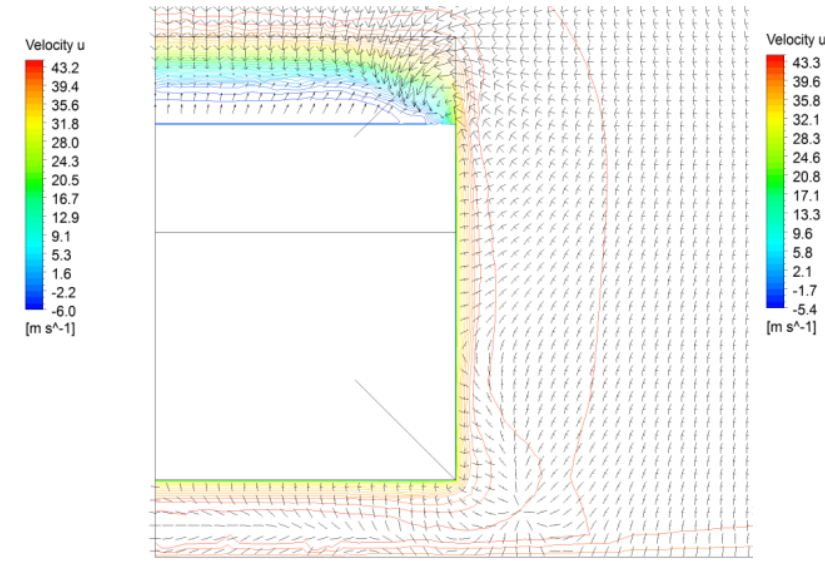

(a) plane $x=-100 \mathrm{~mm}$

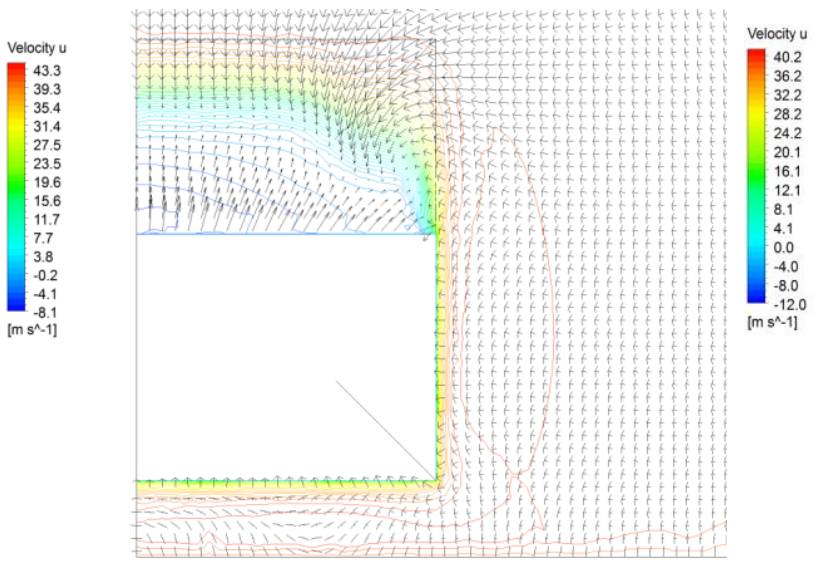

(c) plane $x=0$

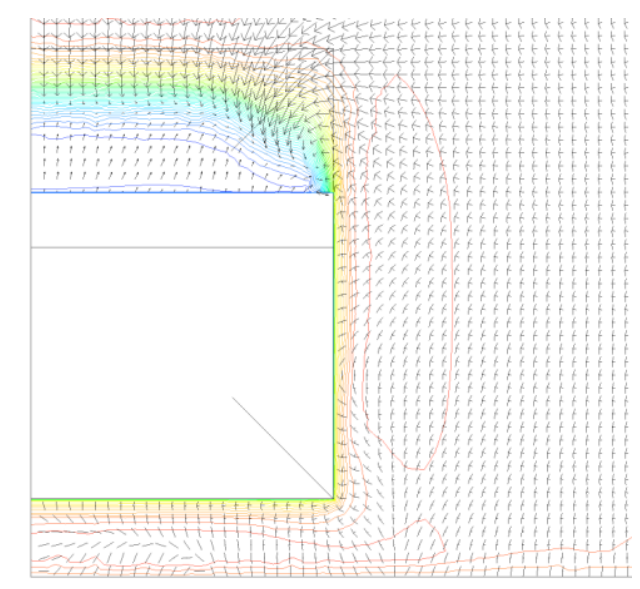

(b) plane $x=-50 \mathrm{~mm}$

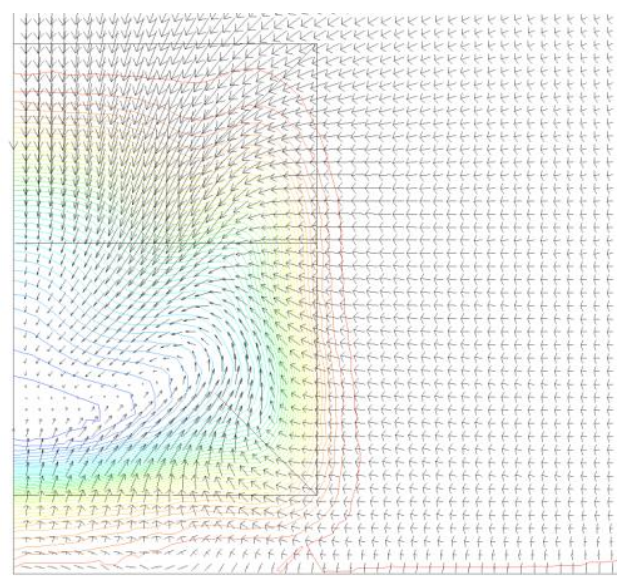

(d) plane $x=0 \mathrm{~mm}$

Figure 4. Velocity vectors and streamwise velocity contour at several planes normal to the streamwise direction. Note that origin $(x=0)$ is located at the leading edge of the spoiler, which is aligned to the rear face of the Ahmed body.

Figure 4 shows the presence of counter-rotating vortices forming from the side edges of the rear slant face on the Ahmed body and trailing towards the rear of the Ahmed body. A similar flow feature was also reported in previous investigations (see for 
example, Figure 11 in ref. [20]), indicating the appropriateness of the present model used in this study.

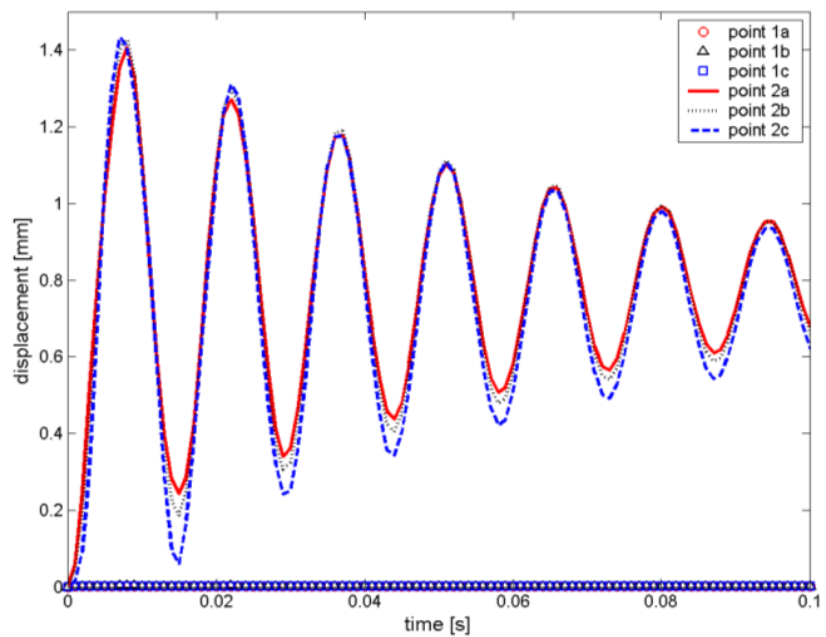

(a)

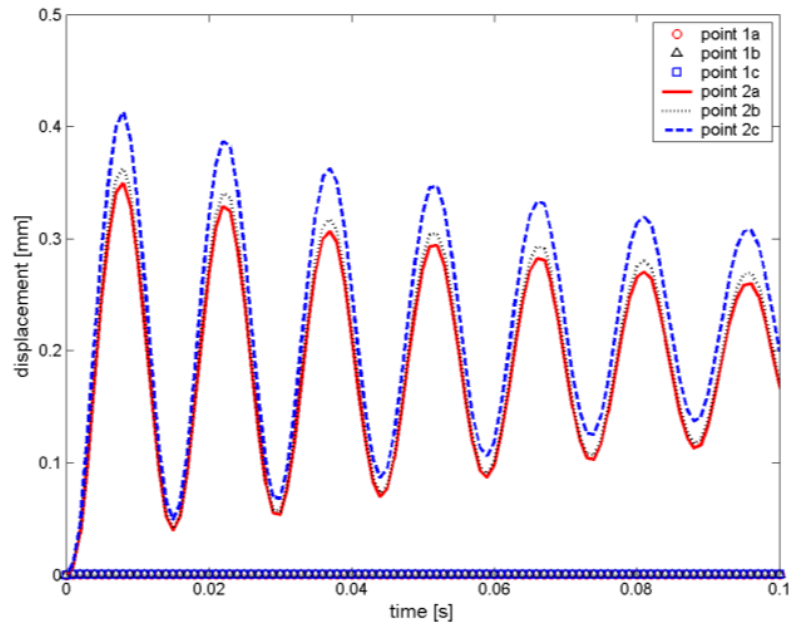

(b)

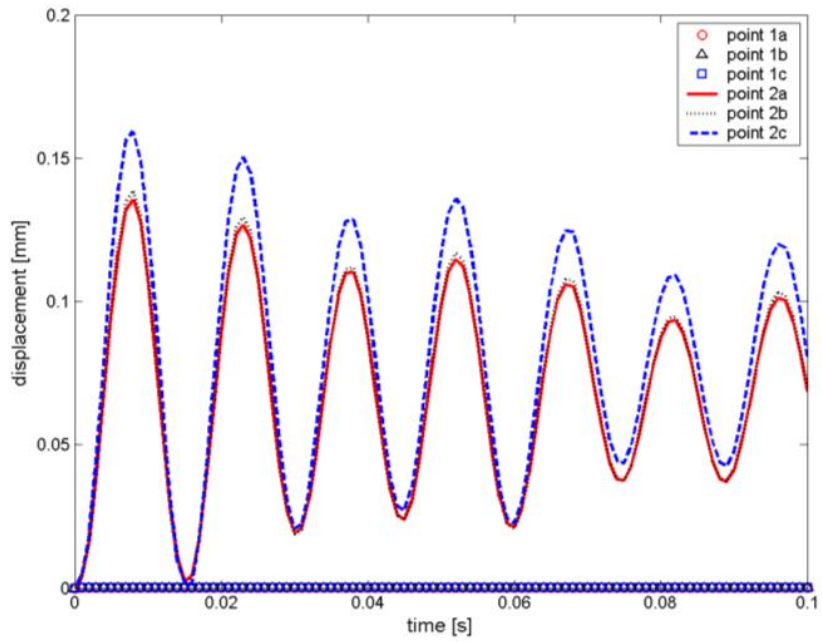

(c)

Figure 5. Displacement history for points near the fixed face and symmetric face (as highlighted in Figure 2) for (a) case 1 (spoiler at $y=0 \mathrm{~mm}$ ); (b) case 2 (spoiler at $y=$ $50 \mathrm{~mm}$ ); (c) case 3 (spoiler at $y=-100 \mathrm{~mm}$ ). 


\section{Rear Spoiler Dynamics}

Three cases that correspond to three different spoiler elevations were simulated. Noting that the origin $(x=0, y=0)$ is aligned with the rear face and the top face of the Ahmed body respectively, case 1 represents a case where the spoiler elevation is exactly aligned with the top face of the Ahmed body (i.e. $y=0$ ). While, cases 2 and 3 respectively represent cases where the spoiler is located $50 \mathrm{~mm}$ and $100 \mathrm{~mm}$ below the top face of the Ahmed body (i.e. $y=-50 \mathrm{~mm}$ and $y=-100 \mathrm{~mm}$ ).

Figure 5 depicts the displacement history of two groups of points i.e. 1a, 1b, 1c and $2 \mathrm{a}, 2 \mathrm{~b}, 2 \mathrm{c}$, that are respectively located close to the fixed face and symmetric face of the spoiler, and where ' $a$ ', ' $b$ ' and 'c' correspond to points at the leading edge, maximum camber and trailing edge, respectively (as highlighted in Figure 2). As expected, the deflections for points $1 \mathrm{a}, 1 \mathrm{~b}$ and $1 \mathrm{c}$, which are very close to the fixed end, are almost negligible compared to the deflections at points $2 a, 2 b$ and $2 c$, which are at the symmetric face and represent maximum deflection zones on the rear spoiler. Figures 5(a)-(c) suggest that as the rear spoiler position is lowered, the maximum deflection of the rear spoiler (which is located on the trailing edge for all cases) is also lowered. In addition, Figures 5(a)-(c) suggest that the amplitude of the rear spoiler vibrations reduces with time until it reaches a steady-state deflection value. However, unlike cases 1 and 2, where the deflection amplitude is continuously reduced, case 3 shows that at instants $t=0.052 \mathrm{~s}$ and $t=0.096 \mathrm{~s}$, the amplitude of the rear spoiler deflection is increased compared to their preceding oscillation.

In the present study, the conversion of mechanical energy to electrical energy may be conveniently implemented using piezoelectric elements or films. Mechanical strain or stress applied on piezoelectric materials leads to the distortion of its dipole crystalline structures, inducing polarization and electrical charge [27]. The distribution of elastic strain on the rear spoiler is plotted in Figure 6, showing the maximum principal strain located close to the fixed end. This may represent an ideal location to place the piezoelectric films in order to optimize energy harvesting.

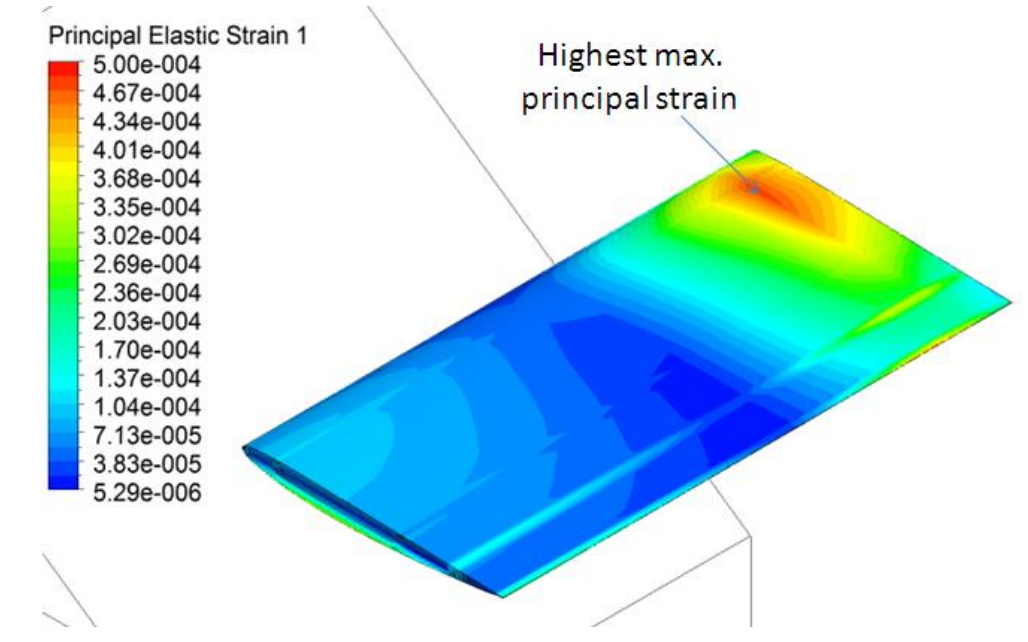

Figure 6. Distribution of the maximum principal strain on rear spoiler.

Figure 7 presents the oscillation of the maximum principal strain over time at the maximum location indicated in Figure 6. Results show that the trend in spoiler principal strain is consistent with the trend in spoiler deflection as highlighted in Figure 5. For case 1 , where the spoiler is located at $y=0$, the maximum principal strain oscillates (at a 
frequency of approximately $70 \mathrm{~Hz}$ ) from a maximum of $610 \mu \varepsilon$ and continuously reduces until an estimated steady state strain of $350 \mu \varepsilon$, as shown in Figure 7(a). While for case 3 , where the spoiler is lowered to $y=-100 \mathrm{~mm}$, Figure 7(b) shows that the maximum principal strain oscillates with a frequency of $60 \mathrm{~Hz}$ from a maximum of $102 \mu \varepsilon$ until it reaches an estimated steady state strain of $52 \mu \varepsilon$. Similar with the deflection history, the maximum principal strain for case 3 indicates that the strain amplitude is increased in comparison to their preceding oscillation, at instants $t=0.052 \mathrm{~s}$ and $t=0.096 \mathrm{~s}$. Therefore, unlike case 1 and case 2, where the strain oscillation is monotonously decreasing over time, case 3 exhibits a certain 'jump' or 're-energized' oscillation at certain instants. This would imply that the total spoiler vibration period for case 3 is expected to last longer than in cases 1 or 2 , before it eventually reaches the steady-state condition.

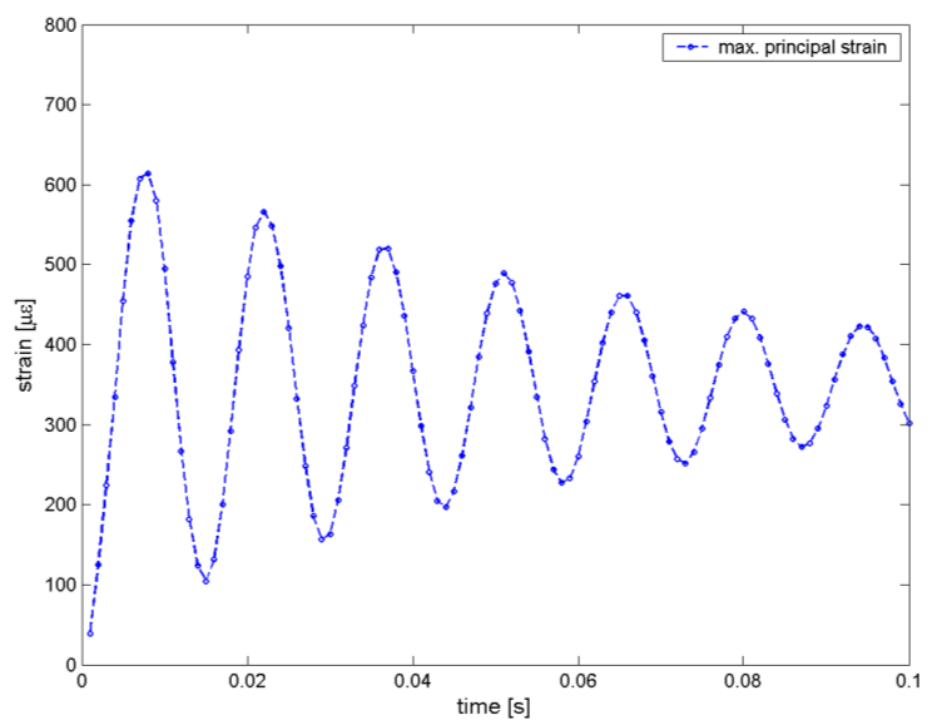

(a)

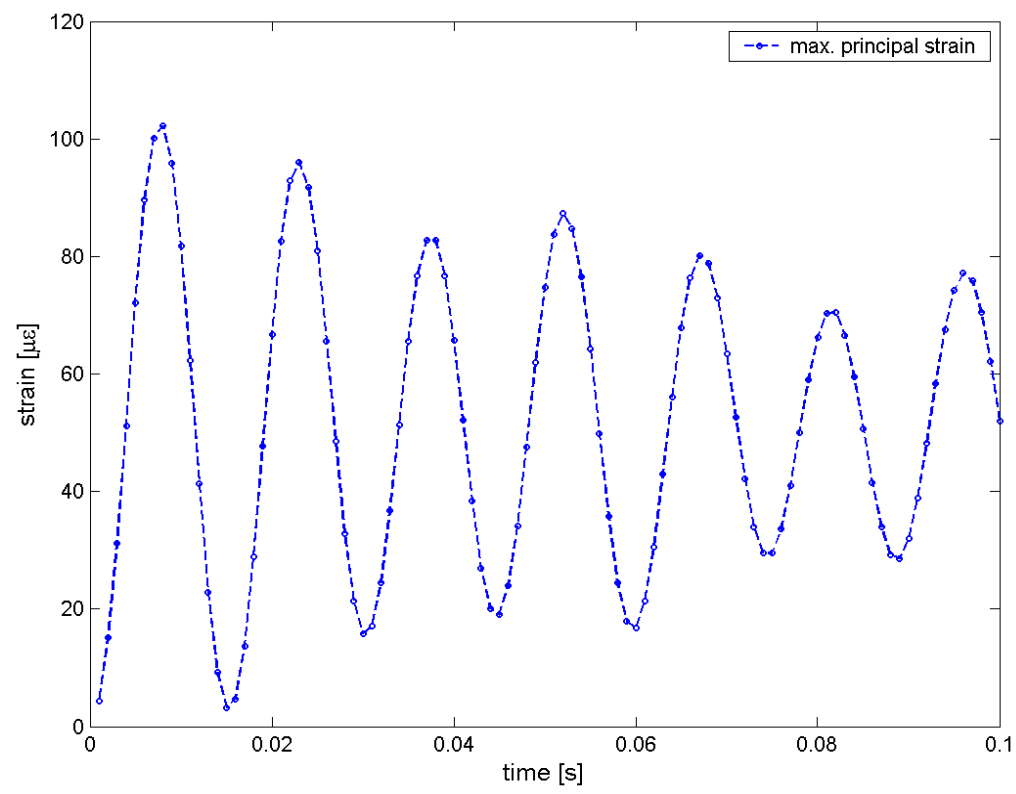

(b)

Figure 7. Maximum principal strain history over time on the rear spoiler for (a) case 1; (b) case 3 . 


\section{Piezoelectric Energy Conversion}

In a previous investigation, Churchill et al. [28] demonstrated the robustness and feasibility of a piezoelectric energy harvester to power a wireless sensor network. A piezoelectric fibre composite under bending strain of $300 \mu \varepsilon$ that cycles at $180 \mathrm{~Hz}$ was reported to output $0.75 \mathrm{~mW}$ of power, while with bending strains of $150 \mu \varepsilon$ at $60 \mathrm{~Hz}$ cycle frequency, $50 \mu \mathrm{W}$ of power was generated (Churchill et al. in [27, 28]). Their finding showed that a minimum $50 \mu \mathrm{W}$ output was sufficient to repeatedly charge a storage capacitor for the successful operation of their wireless transmitter-sensor system, and that the minimum applied strain necessary to achieve this power output increases with the reduction in the oscillating strain frequency.

With an oscillating frequency of $70 \mathrm{~Hz}$, the rear spoiler positioned at highest elevation (case 1) may attain a maximum strain of $610 \mu \varepsilon$ that eventually reduces to a steady-state strain of $350 \mu \varepsilon$, as it interacts with the surrounding flow. This suggests that the minimum power output to power a wireless transmitter could potentially be generated by harvesting vibration energy on a rear spoiler. Furthermore, Figure 7 highlights that as the rear spoiler is positioned lower, its corresponding strain is significantly reduced, but accompanied by a slight reduction in the vibrating frequency (i.e. $70 \mathrm{~Hz}$ in case 1 to 60 $\mathrm{Hz}$ in case 3). This variation of strain with a spoiler elevation indicates that a range of spoiler elevation positions may be identified, where sufficient strain levels for a minimum power output may be harvested.

The conversion of mechanical energy to electrical energy in the piezoelectric is more efficient under vibration compared to the piezoelectric that is statically strained, due to charge leakage. Compared with the strain magnitude in Churchill et al. [27, 28], the piezoelectric on the rear spoiler that is positioned at $y=0$ (case 1 ) is predicted to generate a reasonable amount of energy conversion, although the spoiler vibration period is limited as it monotonously reduces to the steady state. However, with numerous acceleration and stop-start motions during the actual car motion, the spoiler vibration period before reaching the steady state may be longer and sufficient energy conversion may be accumulated during travel. Alternatively, the piezoelectric assembled on rear spoiler that is lowered to $y=-100 \mathrm{~mm}$ (case 3) is expected to vibrate longer before reaching the steady-state and therefore experience an extended period of energy conversion under vibration, although the applied strain and hence expected energy conversion is lower for each strain oscillation. This may be compensated by considering more piezoelectric energy harvesting films or elements to be assembled on both top and bottom surface of the rear spoiler.

\section{CONCLUSIONS}

The flow-induced vibration of a rear spoiler behind an Ahmed body was numerically investigated by coupling an Arbitrary Lagrangian-Eulerian flow solver with a non-linear structural solver. The dynamic response of a rear spoiler at three different elevations was analysed for the energy harvesting potential. In summary, the applied strain on the rear spoiler placed at the highest elevation showed the largest magnitudes. However, the rear spoiler placed at the lowest elevation indicated an extended period of vibration before reaching a steady state condition, as the spoiler oscillation reduces non-monotonically with the presence of an increased oscillation at certain instants.

The numerical results suggest that the rear spoiler vibration may offer sufficient strain levels that could be exploited by piezoelectric materials to harvest ambient energy. For optimum strain energy harvesting, the piezoelectric films or elements are best placed 
at regions close to the fixed or supported end of the spoiler. However, further investigation is required in order to understand those 're-energised' oscillating amplitudes at certain instants when the spoiler is placed at the lowest elevation, and further trials on a car rear spoiler under actual travelling conditions are also necessary to quantify the actual energy conversion.

\section{ACKNOWLEDGEMENTS}

The authors would like to acknowledge the financial support provided by Ministry of Higher Education (project grant code: FRGS/2/2013/TK01/UKM/02/2), and the assistance of resources provided at the facility in Faculty of Engineering and Built Environment, Universiti Kebangsaan Malaysia supported by Dr. Sudarshan Raman.

\section{REFERENCES}

[1] Mohd TAT, Hassan MK, Aziz WA. Mathematical modeling and simulation of an electric vehicle. Journal of Mechanical Engineering and Sciences. 2015;8:131221.

[2] Wahono B, Nur A, Santoso WB, Praptijanto A. A comparison study of rangeextended engines for electric vehicle based on vehicle simulator. Journal of Mechanical Engineering and Sciences. 2016;10:1803-16.

[3] Jiang X, Wang J, Li Y, Li J, Yao J. Energy harvesting for powering wireless sensor networks in low-frequency and large-force environments. Proceedings of the Institution of Mechanical Engineers, Part C: Journal of Mechanical Engineering Science. 2015;229:1953-64.

[4] Akaydin HD, Elvin N, Andreopoulos Y. Energy harvesting from highly unsteady fluid flows using piezoelectric materials. Journal of Intelligent Material Systems and Structures. 2010;21:1263-78.

[5] Weinstein LA, Cacan MR, So PM, Wright PK. Vortex shedding induced energy harvesting from piezoelectric materials in heating, ventilation and air conditioning flows. Smart Materials and Structures. 2012;21, 045003:1-10.

[6] Chan MH. Low-grade waste vibration energy recovery from Hong Kong franchised buses. International Journal of Green Energy. 2014;11:431-7.

[7] Zuo L, Tang X. Large-scale vibration energy harvesting. Journal of Intelligent Material Systems and Structures. 2013;24:1405-30.

[8] Hasan MF, Halim SZ, Shahariar CM, Quddus N. Mitigation of agricultural energy requirement in bangladesh using microalgae production. Journal of Mechanical Engineering and Sciences. 2013;5:646-51.

[9] Khaligh A, Zeng P, Zheng C. Kinetic energy harvesting using piezoelectric and electromagnetic technologies - State of the art. IEEE Transactions on Industrial Electronics. 2010;57:850-60.

[10] Torah R, Glynne-Jones P, Tudor M, Donnell TO, Roy S, Beeby S. Self-powered autonomous wireless sensor node using vibration energy harvesting. Measurement Science and Technology. 2008;19:125202.

[11] Zhang Y, Zheng R, Shimono K, Kaizuka T, Nakano K. Effectiveness testing of a piezoelectric energy harvester for an automobile wheel using stochastic resonance. Sensors. 2016;16:1727. 
[12] Zuo L, Zhang P-S. Energy harvesting, ride comfort, and road handling of regenerative vehicle suspensions. Journal of Vibration and Acoustics. 2013;135:011002-1 - -8.

[13] Tsai C-H, Fu L-M, Tai C-H, Huang Y-L, Leong J-C. Computational aero-acoustic analysis of a passenger car with a rear spoiler. Applied Mathematical Modelling. 2009;33:3661-73.

[14] Kieffer W, Moujaes S, Armbya N. CFD study of section characteristics of Formula Mazda race car wings. Mathematical and Computer Modelling. 2006;43:1275-87.

[15] Jamei S, Maimun A, Azwadi N, Tofa MM, Mansor S, Priyanto A. Ground viscous effect on $3 \mathrm{~d}$ flow structure of a compound wing-in-ground effect. International Journal of Automotive and Mechanical Engineering. 2014;9:1550-63.

[16] Daryakenari B, Abdullah S, Zulkifli R, Sundararajan E, Sood ABM. Numerical study of flow over ahmed body and a road vehicle and the change in aerodynamic characteristics caused by rear spoiler. International Journal of Fluid Mechanics Research. 2013;40:354-72.

[17] Ahmed SR, Ramm G, Faltin G. Some salient features of the time averaged ground vehicle wake. SAE Technical Paper No.840300. 1984.

[18] Hinterberger C, Garcia-Villalba M, Rodi W. Large eddy simulation of flow around the Ahmed body. In: McCallen R, Browand F, Ross J, editors. The Aerodynamics of Heavy Vehicles: Trucks, Buses and Trains (vol 19 Lecture Notes in Applied and Computational Mechanics): Springer Berlin Heidelberg; 2004. p. 77-87.

[19] Guilmineau E. Computational study of flow around a simplified car body. Journal of Wind Engineering and Industrial Aerodynamics. 2008;96:1207-17.

[20] Minguez M, Pasquetti R, Serre E. High-order large-eddy simulation of flow over the "Ahmed body" car model. Physics of Fluids. 2008;20, 095101.

[21] ANSYS Inc. ANSYS CFX Solver Theory Guide - Release 14.52014.

[22] Shih T-H, Liou WW, Shabbir A, Yang Z, Zhu J. A new $k-\varepsilon$ eddy viscosity model for high Reynolds number turbulent flows. Computers Fluids. 1995;24:227-23.

[23] Donea J, Huerta A, Ponthot J-P, Rodriguez-Ferran A. Arbitrary lagrangianeulerian methods. In: Stein E, Borst Rd, Hughes TJR, editors. Encyclopedia of Computational Mechanics: John Wiley \& Sons; 2004.

[24] Kajishima T, Takeuchi S. Simulation of Fluid-Structure Interaction based on an Immersed-Solid Method. Journal of Mechanical Engineering and Sciences. 2013;5:555-61.

[25] Peskin CS. The immersed boundary method. Acta Numerica. 2002;11:479-517.

[26] Kapadia S, Roy S, Vallero M, Wurtzler K, Forsythe J. Detached-eddy simulation over a reference ahmed car model. In: Friedrich R, Geurts BJ, Metais O, editors. Netherlands: Springer; 2003. p. 481-8.

[27] Li H, Tian C, Deng ZD. Energy harvesting from low frequency applications using piezoelectric materials. Applied Physics Reviews. 2014;1, 041301.

[28] Churchill DL, Hamel MJ, Townsend CP, Arms SW. Proc SPIE. 2003;5055:319_ 27. 